\section{IN BRIEF}

\section{TRANSCRIPTION}

Direct observation of DNA rotation during transcription by Escherichia coli RNA polymerase.

Harada, Y. et al. Nature 409, 113-115 (2001)

Several years ago Kazuhiko Kinosita Jr and colleagues used an elegant single-molecule imaging system to show direct rotation of the $\mathrm{F}_{1}$-ATPase. Now they've done it again, this time with a DNAbased motor - RNA polymerase. They show, in real time, that single RNA polymerase molecules attached to a glass surface can rotate DNA for over 100 revolutions, and predict that this technique could help in resolving the individual steps of transcription.

\section{IMMUNOLOGY}

ICOS co-stimulatory receptor is essential for T-cell activation and function.

Dong. C. et al. Nature 409, 97-101 (2001)

ICOS is critical for CD40-mediated antibody class switching.

McAdam, A. J. et al. Nature 409, 102-105 (2001)

ICOS is essential for effective T-helper-cell responses.

Tafuri, A. et al. Nature 409, 105-109 (2001)

Optimal T-cell activation requires an antigen-specific signal as well as a co-stimulatory signal which influences $\mathrm{T}$-cell proliferation, cytokine secretion and the development of effector functions. ICOS is a member of the CD28/CTLA4 family of costimulatory molecules, and is expressed on activated T cells. Its ligand, B7RP$1 / \mathrm{B} 7 \mathrm{H}$, is expressed on $\mathrm{B}$ cells and macrophages. So this pair of molecules does not seem to be involved in initial T-cell activation, but at a later stage during T-cell interactions with B cells and macrophages. To characterize the function of ICOS, three groups have now generated ICOS-deficient mice. They show that ICOS is essential for efficient $\mathrm{T} / \mathrm{B}$-cell interactions and for antibody production in response to T-cell-dependent antigens. Knockout mice have defective germinal centre formation and immunoglobulin class-switching, as well as decreased T-cell production of interleukin-4 and interleukin-13. Further work is required to understand the complex nature of costimulation so that therapies can be designed to treat immune-mediated diseases.

\section{APOPTOSIS}

\section{An alternative, nonapoptotic form of programmed} cell death.

Sperandio, S. et al. Proc. Natl Acad. Sci. USA 97, 14376-14381 (2000)

The terms 'apoptosis' and 'programmed cell death' are often used interchangeably, but this paper describes a form of programmed cell death that fails to fulfil the criteria for apoptosis. Christened paraptosis ('next to' or 'related to' apoptosis), this form of cell death has a distinct morphology and biochemistry to apoptosis, it shows no response to caspase inhibitors or Bcl- $\mathrm{x}_{\mathrm{L}}$, and it is mediated by an Apaf-1-independent caspase- 9 activity.

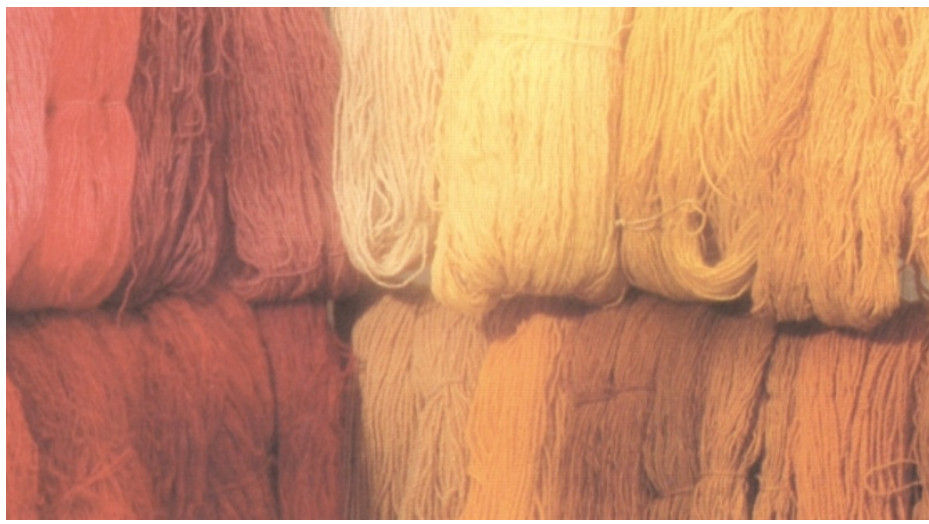

CHOLESTEROL BIOSYNTHESIS

\title{
Unpicking NPC
}

Niemann-Pick disease type $\mathrm{C}$ is a hereditary disorder that leads to progressive neurodegeneration and death during early childhood. More than $95 \%$ of patients have defects in the NPC1 gene, whereas a small subset of patients have defects in a second gene, NPC2. But in both cases, cholesterol accumulates aberrantly in the lysosomes. Two reports in Science now bring us a step closer to understanding the processes behind both forms of this debilitating condition.

Yiannis Ioannou and colleagues investigated the function of the protein that is mutated in NPC1 disease by looking for conserved motifs in its sequence. They found that it contains six copies of a lipid-attachment site also found in members of the resistance-nodulation-division (RND) family of prokaryotic permeases. These proteins are involved in pumping hydrophobic compounds such as antibiotic drugs, detergents and fatty acids from the cytosol of Gram-negative bacteria.

Two members of the RND family - Escherichia coli AcrB and Pseudomonas aeruginosa MexD show weak sequence homology to the NPC1 protein throughout their entire sequences. Moreover, NPC1 and MexD have similar membrane topology and secondary structures. So could human NPC1 be an RND permease? Ioannou and colleagues tested this by looking at accumulation of a fluorescent dye called acriflavine in normal and NPC1-deficient fibroblasts. They found that efflux of acriflavine from the endoso$\mathrm{mal} /$ lysosomal system is blocked in the absence of NPC1, and that this efflux is an active process, requiring a proton-motive force.

Next the authors engineered $E$. coli to express human NPC1. Then, given that the defect in NPC1 disease is the accumulation of cholesterol, they wondered whether this might be a substrate for NPC1. Ioannou and colleagues could observe no build-up of cholesterol in NPC1-expressing E. coli cells, but they did see an accumulation of oleic acid indicating that, like its prokaryotic homologues, NPC1 might transport fatty acids across a membrane.

In the second paper, Naureckiene et al. describe the protein responsible for NPC2. While trying to characterize the lysosome proteome they identified a human protein called HE1. The pig homologue of this protein is known to bind cholesterol so, given its lysosomal location, the authors wondered whether HE1 might be involved in NPC2 disease. HE1 could not be detected by Western blotting in fibroblasts from two patients with the condition, and sequence analysis revealed that both patients had mutations in the HE1 gene.

Studies with a cholesterol-binding antibiotic, filipin, indicated the abnormal accumulation of cholesterol in fibroblasts from the NPC2 patients. But when Naureckiene and co-workers treated these fibroblasts with recombinant $\mathrm{HE} 1$, this build-up was reduced. The HE1-conditioned medium had no effect on cholesterol accumulation in NPC1-deficient fibroblasts, however, confirming that the defects in the two forms of NPC disease are different.

6) References and links Alison Mitchell ORIGINAL RESEARCH PAPERS Davies, J. P., Chen, F. W. \& loannou, Y. A. Transmembrane molecular pump activity of Niemann-Pick C1 protein. Science 290, 2295-2298 (2000) Naureckiene, S. et al. Identification of HE1 as the second gene of Niemann-Pick C disease. Science 290, 2298-2301 (2000)

FURTHER READING Marx, J. Disease genes clarify cholesterol trafficking. Science $\mathbf{2 9 0}$ 2227-2229 (2000) 